\title{
Diplopedia Imagined: Building State's Diplomacy Wiki
}

\author{
Chris Bronk \\ Rice University \\ rcbronk@rice.edu
}

\author{
Tiffany Smith \\ U.S. Dept. of State \\ smithtl7@state.gov
}

\begin{abstract}
Considerable interest has been directed by the Obama Administration in harnessing Web 2.0 technologies such as blogs, wikis, social media and cloud computing to overhaul the business of government. Addressed here is the account of an enterprise wiki at the U.S. State Department. That wiki, Diplopedia is currently employed by the State's employees to share subject matter knowledge related to the process of diplomacy. Contained herein is an account of how Diplopedia was conceived and became a functional system for knowledge sharing at the Department of State (DoS).
\end{abstract}

KEYWORDS: e-government, wikis, Web 2.0, social software, digital diplomacy, enterprise architecture.

\section{INTRODUCTION}

THIS paper is an account of how the State Department's Office of eDiplomacy deployed an enterprise wiki knowledge repository and the open source software required for it to run. Scholarly interest in Wikipedia and the act of collaborative knowledge construction has grown rapidly over the past several years. There is considerable interest in the adaptation of wiki and other social software technologies in a reinvention of government, often labeled Government 2.0. The authors' notes, recollections and research for this paper aim to fill some small piece of the knowledge gap that resides between vision for employing social software in government and the realities of what is required to begin making such software a formal component of the process of government.

There are two major topics addressed drawing on the process to deploy the State Department's Diplopedia enterprise wiki: (1) how a government agency came to accept the potential utility of a wiki platform for knowledge construction and dissemination; and (2) the steps required to integrate open source software into that agency. This includes one author's account of the process undertaken during his tenure as a Foreign Service business practice advisor to the Office of eDiplomacy during 2005 and 2006. The second author, a current employee of eDiplomacy, served as Project Lead for Diplopedia from 2007 to 2009, and provides further information on the adoption of the wiki's use by personnel during that time and beyond. The story begins with a visit from Jimmy Wales, Wikipedia's "God-King" and chief booster.

\section{THE IDEA - MEETING MR. JIMMY}

As unlikely as it sounds, on the morning of April 19, 2006, Jimmy Wales, guiding light of Wikipedia, addressed participants attending the Intelink Technical Exchange. Wales delivered a single message arguing that the participants in the Wikipedia experiment were grown-ups and could be trusted to do the right thing in entering their knowledge into the encyclopedia. By extension, Intelink's customers, the intelligence analysts and their managers who supplied assessment to policy-makers might also be entrusted to use the wiki model to develop their assessments in a collaborative fashion. Already, individuals in the Intelligence Community (IC) had begun to think about the new technologies turning up on the Internet, including blogs, wikis and other tools considered to fall under the heading of collaboration software.

In 2004, the CIA's Calvin Andrus won the Galileo Award for his paper, "The Wiki and the Blog," on the value of Web 2.0 technologies to the business of intelligence [1]. Another Galileo paper, Matthew Burton's "Connecting the Virtual Dots: How the Web Can Relieve the Information Glut and Get Us Talking to Each Other," attacked the same problem set, but from a different tack [2]. Andrus's approach spoke to his background as a political scientist while Burton's took a tone cohering more with the Silicon Valley mindset, but both clearly stated that new paths to organizational performance in intelligence existed by the employment of the latest generation of IT.

\subsection{A Diplomacy Wiki}


By 2005, a number of government IT staffers around the U.S. federal bureaucracy had been touched by the idea of the wiki. To those who had worked in software development, wiki software brought to the world of text what had long existed in software code: the ability to track who contributed what. While much software has been and is still written by solo practitioners, software tools for merging the efforts of many have been around at least since the 1980s. The key take-away was the provision for version or revision control.

This notion of version control was embedded in the functionality of MediaWiki, the software driving Wikipedia. It allowed many individuals to contribute, whether in fleshing out the subject matter of an article, linking to related materials or improving the prose. ${ }^{1}$ Each change could be attributed to a contributor's user identity or the Internet Protocol (IP) address of the computer from which the change was made. This made it easy to assess and compare who said what and when. Wikipedia allowed multiple authors to work on a document, contributing their expertise and leaving to someone else to fill in what they did not know.

Inside government, wiki software offered tremendous capabilities for the horizontal sharing of analytical information. At the State Department, entropy, framed in the semantic interpretation of the term found in information theory [3], was the problem. Foreign Service Officers (FSOs), who move around the globe were expected to acquire a degree of expertise rapidly in each new job, but upon leaving the job, this knowledge could be lost. The organization did not have a strong system where prior job incumbents could be called upon to explain the intricacies of job process or subject matter.

This entropy problem of an organization susceptible to knowledge loss was recognized as a key issue to the DoS's Office of eDiplomacy, who recognized that "How to?" questions were among the most frequently asked, especially by junior staff. Above all else, the U.S. State Department is charged with crafting foreign policy. Drawing upon the reporting of its constellation of diplomatic missions around the globe, the State Department's primary task is to combine multiple information streams to craft policy for bilateral and multilateral diplomatic initiatives.

Still, the preferred medium of official communication from mission to mission remains an amalgam of the telegram. Criticism remains of the State Department's capacity to cope with the digital world it inhabited. As one FSO stated, "We don't train people to disseminate information properly. We should be looking at training so that people know how to make their documents accessible to everyone who might want to use them-whether that is via a listserv, wiki, blog, or e-mail" [4].

To many in the State Department, it remained unclear where their reporting from overseas missions went in Washington. A system of conventions regarding tagging and delivery of telegrams exists in the Foreign Affairs Handbook [5]; however, matching producers of reporting with consumers was an exercise in learning the lay of the organization, which might take months or years of experience.

\subsection{Getting SMART about Collaboration}

While eDiplomacy thought about what a diplomacy wiki might look like, the State Department was in the process of updating its telegram system through a project called SMART - the State Messaging and Archive Retrieval Toolset. SMART was emblematic of large project IT, designed to meet exhaustive technical specifications and also comply with extensive federal requirements. Acting CIO Jay Anania summed up the project and also gave hint to just how rapidly the requirement set for enterprise software was changing. On SMART, he said, "This is an extremely important effort that will replace our legacy telegram system used for command, control and communication ... to share information and promote knowledge management. The vision is that we'd also have additional functionalities [such as] collaboration software." [6]

Anania helmed IRM as Web 2.0 began influencing government. Web 2.0 seemed like one part of a larger answer to the information sharing problems raised by members of the 9/11 Commission, but actually deploying them in a government agency was confounded by their newness. Collaboration was a hot topic in 2005; however, visions of how to operationalize the term remained fuzzy. A diplomacy wiki was one of several potential collaboration options on the table in 2005. The question was how to gain visibility and legitimacy for such an idea.

\subsection{Learning from Communities}

Of fundamental importance in getting a diplomacy wiki running was the need to establish clear mechanisms for the governance of content creation and conflict resolution. Fortunately, Web 2.0 governance at the State Department had already been considered by those managing eDiplomacy's Communities@ State (C@S) initiative. Harnessing advice from productivity consultancy APQC,

\footnotetext{
${ }^{1}$ It is wise to set aside issues of correct or incorrect, right or wrong, for wikis at this point in the paper.
} 
Open Source Intelligence System (OSIS) ${ }^{2}$ hosted blogs were established as community of interest/practice web hubs. The goal for C@S was to form communities of practice surrounding a certain topic, process, or domain of knowledge. These blogs, indexed by a search appliance, would be easy to find, and those with the greatest expertise would be able to contribute their knowledge in a horizontal manner.

Rather rapidly, a number of interested groups came forward willing to try the new technology. Early successes were driven by a number of factors. Consulate General Chengdu's "Chengdu News" had the backing of a technically savvy officer at post, who also had an interesting topic to investigate, China's continuing efforts to control avian influenza. Other $\mathrm{C} @ \mathrm{~S}$ blogs were a liaison device between individuals who were solo practitioners or small sub-groups: for instance human rights reporters or marine security guards. ${ }^{3}$

In blogs, eDiplomacy's leadership had an emerging success in its portfolio with the C@S, but an enterprise wiki would have much more visibility, and with it a higher level of potential risk. The vandalizing of John Seigenthaler's Wikipedia biographical entry, labeling him as a conspirator in the assassinations of John and Robert Kennedy [7], gave reason for concern that Wikipedia might be too loosely run to serve as a model for the State Department or any other government agency.

\section{MOBILIZING TRANSFORM-ATIONAL DIPLOMACY}

By the beginning of 2006, Secretary of State Condoleezza Rice's January 18 speech at Georgetown University would give some indicator of how well the State Department's leadership had taken technology issues to heart [8]. While leaders seemed pleased with their BlackBerrys, it was an unknown if any other digital initiatives, such as wikis, blogs, and Virtual Presence Posts, were on their radar.

The issue that grabbed the speechwriters' attention for the historic transformational diplomacy address was virtual presence. Secretary Rice homed in on that topic as her transformational technology element, stating:

Perhaps the newest and most cost effective way to adopt a more local posture is through a Virtual Presence Post. Here one or more of our young officers creates and manages an

\footnotetext{
${ }^{2}$ In this case, the "open source" in OSIS refers to openly available, i.e. unclassified sources of information relevant to the business of intelligence.

${ }^{3}$ Over time, some of the communities disappeared, but Chengdu News celebrated its $5^{\text {th }}$ anniversary in January 2010.
}

Internet site that is focused on key population centers. This digital meeting room enables foreign citizens, young people most of all, to engage online with American diplomats who could be hundreds of miles away. This is a great way to connect with millions of new people across Europe and Asia and Latin America [9].

With the mention of the Virtual Presence Post, Rice had taken an eDiplomacy idea as one of importance to the State Department. Although eDiplomacy was a small operation, the speech had marked it as a shop with usable ideas. This would allow the office's staffers to employ transformational diplomacy as a tool for bringing its ideas into action. A certain degree of cover had been granted and a window opened. While there was friction on how and what should be done next, it was around this time that dialog beyond the boundaries of the State Department came to the fore.

\subsection{Getting out the Door}

The MediaWiki application falls into the category of free or open source software. The Wikimedia Foundation, the non-profit that runs Wikipedia and manages the evolution of the MediaWiki software does not charge for what it makes, but rather runs on philanthropic donations and volunteer labor. It is the sort of activity that deeply confuses economists [10].

Up until 2006, the DoS bought software, labeled proprietary, produced by major software firms, and then adapted to the missions and processes of the organization and those responsible for IT at the State Department, primarily its Bureau of Information Resource Management (IRM). To introduce new software to the State Department's networks, a change control process was instituted to bring under control the configuration and management of multiple software installations to comply with federal statute [11]. On October 23, 2001 the State Department Information Technology-Change Control Board (IT-CCB) was formed. It was established to set a baseline for determining what software and hardware could be connected to the network and how. An overarching concern was the problem of configuration management [12]. . This was clearly highlighted in the Foreign Affairs Manual chapter regarding information systems management.

Configuration management provides assurance that an information system in the operational/maintenance phase of its lifecycle is the correct configuration and that any changes made are reviewed for security ramifications prior to implementation. Configuration management ensures that changes take place in an identifiable and controlled fashion and have been tested to preclude adverse affects on the information system's functionality, including its security 
posture or any connecting Department information system's security posture [13].

Without IT-CCB approval, no new piece of software could be added to State's enterprise network. The process of bringing to a vote the provision of servers capable of running the MediaWiki software required a plan for developing consensus among stakeholders who did not always agree with one another. To make it to a vote, a lengthy checklist of preconditions needed to be met by proponents of wiki software at the State Department.

Among the requirements for the State Department's wiki software was compliance with Section 508 of the Americans with Disabilities Act (ADA). For the larger players in the federal IT market, 508 compliance was a given, with many providing the requisite paperwork, the Voluntary Product Accessibility Template (VPAT). ${ }^{4}$ While many items needed to be addressed before MediaWiki could be run in the DoS's data center, getting the VPAT became an early target and a reason to reach out to the Wikimedia Foundation.

Wikimedia's legal counsel fielded a call from an eDiplomacy staffer regarding the foundation's willingness to provide a VPAT. While there were some details to be hashed out, the answer was a tentative affirmative. A series of correspondences ensued, and eventually a MediaWiki VPAT was completed. It also came with a request. The legal counsel and his boss, Jimmy Wales, asked if someone could speak about the diplomacy wiki at the next meeting of the Wikimedia Foundation, Wikimania, scheduled for August 2006.

\subsection{Pilot}

While VPAT was no longer an issue, the other requirements for bringing MediaWiki and its associated pieces of open source software into the State Department enterprise network seemed insurmountable. In the spring of 2006, the Office of eDiplomacy changed leadership to a Civil Service deputy director able to make inroads in the DC bureaucracy of the State Department, and a Foreign Service director capable of working the grapevine, meant that eDiplomacy was properly staffed at the top to achieve results.

In moving forward, eDiplomacy staff debated combining the Communities@ State and Diplopedia initiatives in some way. From a technical standpoint, the two shared essentially the same web server infrastructure, the LAMP stack (Linux-Apache-MySQL-PHP). In 2005, all four

\footnotetext{
${ }^{4}$ Software developer Adobe provides VPAT documents via a clearly organized website. See the Adobe Acrobat 8 VPAT,

http://www.adobe.com/products/acrobat/pdfs/Acrobat_8_VPAT.pdf.
}

were clearly in the domain of open source software. ${ }^{5}$ What Movable Type and MediaWiki held in common was that both needed PHP, the scripting software employed to dynamically create web pages, and a database, preferably MySQL, to hold the content repositories of information stored by both applications.

MediaWiki and Movable Type were bundled together in a single IT-CCB submission labeled lightweight collaboration technologies. There were plenty of "heavy" collaboration technologies being evaluated, but each needed a large piece of software to be installed and run on each computer on which it was to be used. The required software to use MediaWiki was an Internet browser. To the diplomacy wiki users, especially those interested in using it as an information resource and not contributing to it, it would be just another web site.

With the lightweight collaboration technologies suite well defined, the issue shifted to actually getting the two applications and the LAMP stack onto real servers in the data center. In meetings with IRM/Operations, the notion of deploying Linux, an open source operating system, was considered deeply problematic. The State Department had minimal experience with Linux and its information security officers knew that certifying a new operating system for enterprise use would be a monumental task.

In response to the LAMP request, a compromise was offered by a technical analyst in IRM's Information Assurance (IA) office. Linux was a "no-go" as the process for clearing a new operating system for operation on State's unclassified OpenNet network would likely take months. Instead MediaWiki and Movable Type had to run on a Windows "box" and IA would be willing to support a pilot program of the two applications. Although "L" and "A" were out of the mix, "M" and "P" were acceptable. IRM/IA would follow up by performing an extensive set of penetration tests.

Helpful in the process of moving toward deployment was the discovery of the Inter-Agency Wiki Technical Engineering Group. Composed of representatives from the IC, and managed by MITRE, the Inter-Agency Wiki Group was an important window into the change in culture regarding the sharing of information in the post9/11 IC. Conversation alternated between particulars on authentication to strategies for getting buy-in from higherups. It was here that eDiplomacy's staff learned that the IC's wiki, Intellipedia, was really going to happen. The DNI's Chief Information Officer, Air Force General Dale Meyerrose, was firmly behind it [14].

\footnotetext{
${ }^{5}$ MySQL was later acquired by Sun Microsystems, which, in turn was acquired by database giant, Oracle Corporation. Oracle has, thus far, pledged to continue development of MySQL as a competitive product.
} 


\subsection{Intellipedia's Unclassified Cousin}

While the IRM/Operations cohort was understandably nervous about installing software that could compromise the network, a security audit of the servers completed by IRM/IA was successful. All of the pieces worked together and penetration testing yielded no major faults. The lightweight collaboration technologies pilot was moving along smoothly.

Throughout 2006, questions lingered about the wiki, not least what to call it. If diplomacy was to have a wiki, why not call it Diplopedia? ${ }^{6}$ Suddenly State's wiki seemed a great deal more real. A wiki would be created. It would be an internal State Department resource. It would provide information of importance in the conduct of diplomacy, and it would be unclassified.

An iron clad governance structure was needed for what could conceivably grow to be a large and vital information resource for the State Department. Obviously, Wikipedia editors disagreed from time to time, so what did the free encyclopedia offer as its type level guidance? When pressed, the reply was offered, "the Wikipedia people seem to say, 'Don't be a jerk." While such a coarse statement would not work as a governance norm for a government agency, "Assume the good intentions of others," was an acceptable substitute. The additional points for good Diplopedia behavior were largely borrowed from Wikipedia as well, including:

- If something is wrong, change it.

- If something is missing, add it.

- Use plain language.

- Use the "Discussion" tab to discuss an article.

- Use neutral point of view.

A final incentive for good etiquette in Diplopedia was enforced by the policy regarding anonymous edits. Although Wikipedia permitted individuals to edit articles without logging into it (and still does so for less contentious articles), anonymous editing of a State Department information resource was unacceptable. To edit Diplopedia, users would need an account, which leveraged their existing network identity. Every contribution would link back not to an obscure Internet Protocol address, but to a real State Department employee. Accepting that any exercise in the anticipation of unexpected behaviors would be incomplete at best, other governance issues were to be sorted out as they emerged.

\subsection{Wikimania}

\footnotetext{
${ }^{6}$ Concerns of confusion with diplopodia, a medical condition in which
} the subject possesses an additional foot, were raised, but soon tabled.
Early in eDiplomacy's discussions with the Wikimedia Foundation's general counsel, the invitation was offered for a State Department representative to speak at Wikimania, the foundation's annual conference, held August 4-6, 2006 at Harvard. For the conference, Diplopedia's business case was explicated in the following manner:

America's diplomats are tasked with sending from overseas embassies and consulates reporting laden with added value beyond what may be read, seen, or heard in open sources by policymakers at home. To be effective, today's diplomat must convey a deeper understanding of his or her country beyond the international headlines. A contextually rich view of issues, infused with comments produced by conversations with government ministers, academics, and taxi drivers, is the desired deliverable to the institution. The information revolution brought to an end the summarizing by diplomats of the local news to Washington, however, it now permits individuals to collaborate electronically at global distances on shaping the perception of issues, trends, and events. A profoundly hierarchical organization, the Department of State's employees have engaged in collaborative narration for creating official reporting, with junior-level drafters producing documents for mid- and senior-level editors and approvers. Beyond this formal messaging between diplomatic posts and Washington has proliferated heavy traffic of horizontal messages delivered by email and web pages. While this communication has been a boon for the sharing of knowledge, it is haphazard and uneven in its delivery of desired content to intended recipients [15].

Wikimania 2006 drew heavily among those doing the work of Wikipedia, editing its articles, putting together the technical infrastructure to run it and managing MediaWiki's code. For some, it was an opportunity to get excited about software again, an afterimage of the late 1990s Internet boom. Others were drawn by the altruism of the free knowledge movement, which had longstanding roots in the GNU community shepherded by Richard Stallman at MIT.

Also present was a different open source crowd, those working in the field of Open Source Intelligence. Highly visible was Robert Steele [16]. A forceful critic of the IC's culture of secrecy, Steele had argued that with the Internet, much of the intelligence business could be radically re-thought based upon the proliferation of open sources available via the Web [17]. Leadership in the Office of the Director of National Intelligence (ODNI) was coming around to the idea that open source software and open source intelligence could be a powerful recipe for intelligence collection and analysis. ${ }^{7}$

\footnotetext{
${ }^{7}$ Note: The author recently discussed Wikimania with former Office of the Director of National Intelligence Chief Information Officer retired Gen. Dale Meyerrose. "Yeah, I was there," Meyerrose admitted. Now a
} 
What the State Department got from Wikimania was internal credibility. Staffers from eDiplomacy went to Harvard, talked about Diplopedia and nothing bad happened. After months of an organizational struggle in which barriers often seemed insurmountable, the possibility of an enterprise wiki running at the State Department seemed more likely. Upon returning to Washington, a representative from IRM's data center asked if employing virtual servers to host Diplopedia and the blogs was an option. Making the first deployment of open source software at the State Department also employ the first virtual machine seemed a natural fit.

\subsection{Deployment}

In the September 2006 meeting of the State Department IT-CCB, the deputy director of the Office of eDiplomacy nominated the Lightweight Collaboration Tools pilot for a vote. The major issue for the pilot, systems security, was addressed by IRM/IA's analyst. Both IRM/IA and Diplomatic Security's CTAD agreed that there was no undue risk in deploying MediaWiki or any of the other software. After brief discussion of the project lead by the board's chair, the matter was taken to a vote. The bureau representatives were unanimous in their approval.

With pilot approval, hardware was provisioned, virtual servers initialized and software installed. Within a few weeks, the Diplopedia wiki was created, still holding the MediaWiki "look and feel" but skinned with a DoS graphics scheme and its permissions set to meet the policies for the wiki, chiefly that no anonymous edits would be permitted. The wiki was internally-facing and permitted edits only by those with access to the State Department's unclassified OpenNet network.

In building out a wiki for a government agency, it is natural to assume that all sorts of special software tweaks would be necessary to meet government specifications. With regard to Diplopedia, this is not the case. Let it be clearly stated here that no special wiki software was needed for this effort, nor was any alteration of the MediaWiki source code conducted by the State Department. While the Microsoft Windows Server-IIS platform approach was unusual, it still involved no inhouse software development, but rather integration of commercial off-the-shelf or open source software components.

Tremendously useful was advice from a member of the Inter-Agency Wiki Technical Engineering Group regarding the alteration of MediaWiki's source code, who argued, "You don't want to fork the code." Forking, the

computer security executive for Harris Corporation, Meyerrose was the driver for constructing the IC's Intellipedia wikis. process of making changes that would make the version of MediaWiki different than what the Wikimedia Foundation's development cadre and community of volunteers produced, was be a mistake. After making changes to MediaWiki to meet the technical and policy requirements of his agency, it was no longer compatible with new releases of the software. Taking this advice, a stable full release of MediaWiki was selected and deployed sans tweaks.

\section{WELCOME TO DIPLOPEDIA - CURRENTLY, THERE ARE 10,096 ARTICLES}

Diplopedia was installed on May 26, 2006, and launched on September 26, 2006. In just over three years, Diplopedia has grown from a tiny database of about ten seedling articles to more than 10,000 articles as of January 2010 [18]. While the size of the initial repository was small, its content was created by State Department employees. In following this strategy, another lesson taken from the Intellipedia architects was applied: Do not pull Wikipedia articles as content for Diplopedia. While it seemed like a good idea to pull in pages from Wikipedia as templates, such an effort flew in the face of why and how users in government would want to use Wikipedia and Diplopedia. While it would be slow process to generate content, "slurping" it from Wikipedia was the wrong way to start.

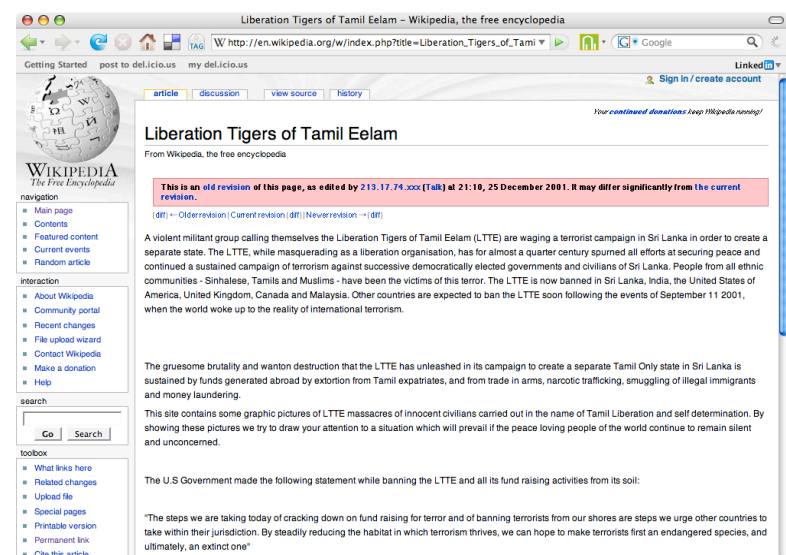

Figure 1. First version of LTTE page in Wikipedia, December 2001

Figures 1 and 2 show how Wikipedia articles improve in quality over time because multiple editors make contributions on the topic. They display the first instance of the Liberation Tigers of Tamil Eelam indicate the organic growth and organization that takes place in a Wikipedia article. Hauling an article off to a secure network cuts it off from the primary contributors who 
improve it. ${ }^{8}$ The first paragraph in the About Diplopedia article has changed very little in three years.

Diplopedia is an online encyclopedia of foreign affairs information. It is a wiki, a kind of Web site that can be edited with an OpenNet Web browser. Subject to the principles and guidelines for Diplopedia, anyone who can access Diplopedia is invited and encouraged to contribute his or her experience, knowledge and expertise in the form of articles, discussion or editing of material submitted by others. It is fast becoming a reference and starting point for all topics of interest to the Department and USG foreign affairs community. [19]

The only sentence that has changed since September 2006 in the paragraph is the final one. Originally, it read "Eventually, eDiplomacy would like it to be a reference and starting point for all topics of interest to the Department." This is significant for two reasons: the first is that instead of implying a yearning, the sentence states something that is occurring, desirous or not. Second, the foreign affairs community was brought to the table, and the fact that Diplopedia is made available to many colleagues outside of the firewall was honored.

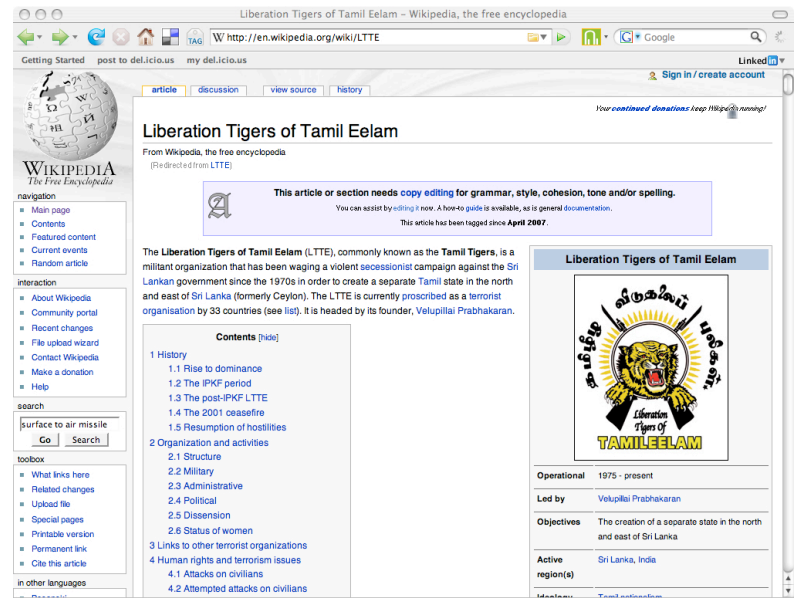

Figure 2. Wikipedia LTTE page, July 23, 2007.

These changes are symbolic of others that occurred over the course of three years of collaboration and led to its prominence as a reliable resource for foreign affairs information. On the enterprise level, Diplopedia in some ways followed the Wikipedia model [20], but in other ways the information contained in Diplopedia has been more stable through time, perhaps because of the limited number of editors and audience. The collaborative editing possibilities for Diplopedia, combined with the reliability of the editor identities, created an environment that allowed Diplopedia to flourish while limiting inquiries about its viability.

\footnotetext{
${ }^{8}$ Although this does beg the question of which Intellipedians or Diplopedians are avid Wikipedians as well.
}

\subsection{What's Different and What's the Same}

Whereas the Open Source culture itself is often cited as one of the reasons for Wikipedia's success, the DoS, as one might expect, does not have a strong Open Source culture. Success in a diplomatic career on a personal level is not traditionally derived from a collaborative and creative professional milieu; instead, it comes from many years of successfully analyzing and acting upon information from disparate sources, and communication is rarely distributed across organizational boundaries [21]. Despite this, motivation exists for finding, identifying, and collecting data, and Diplopedia's success is in many ways attributable to providing answers as needed to "active lurkers."

"Active Lurkers" are those groups that gather value from a social media resource without directly contributing to it. They also tend to promote the resource to others [22]. This is consistent with the general 90-9-1 theory of Participation Inequality for Web engagement, derived from the 80-20 rule or Pareto principle [23]: generally, collaborative software serves as a reference source for the majority of its audience, a small percentage contributes to it, and a very small percentage actively contributes. Both from anecdotal evidence and by looking at the growth statistics outlined below, it is clear that active lurkers were at work within the Department.

Another principle observed behind the firewall was wiki neutrality. Neutral point of view, while often actively moderated on Wikipedia, has not been an issue for Diplopedia. In part, this could be due to the diplomatic culture (employees regularly use the passive voice in their writing), or due to the lack of anonymity that can protect those Wikipedians from repercussions. There is no incentive to deface an article, or speculate on a topic with which you're not familiar, if the wiki reflects your thoughts and work.

The concept of low barriers to entry is a great draw for Department staff: as with Wikipedia, they can create a login in under sixty seconds. In an environment where travel form processing can take the better part of a workday, having the ability to correct a typographical error on a page without having a supervisor sign off on changes.

One of the initial realizations that occurred to staff dedicated to this project was that there wasn't a need to teach the Open Source ideals. Even training employees to understand what a wiki is wasn't an effective use of time. Instead, it was important to demonstrate how Diplopedia could fill immediate needs and help solve longstanding business process issues. 


\subsection{Content Is King}

One of the first breakthroughs in widespread adoption of Diplopedia was Deskipedia. Deskipedia originated as a Community@State, the multiauthor blogging platform. It was designed to help new desk officers, who serve as the conduit of information between Washington and overseas posts. Information about the country that a desk officer covers is paramount in the position, but a successful incumbent must know jargon, write Daily Activity Reports, get policy cleared by appropriate staff and offices, and deliver more mundane items like requesting motor vehicle transportation. All of these processes are relatively consistent, no matter the country or organization. Deskipedia provided a central location for information on how to complete this work efficiently and correctly

Such a service didn't require a lot of discussion, and the time-centric information organization of a typical blog didn't make it easier for personnel to search the database. After Diplopedia was available, moving Deskipedia into it was a natural fit. Through a wiki, officers could add and update information on procedures that might have changed since a blog post was originally written, and they could also network topics together in order to further define information.

The task of migrating the Deskipedia blog to the Deskipedia portal in Diplopedia was left to eDiplomacy staff and wiki enthusiasts throughout the Department, who were interested in using this example as the proof of concept for how information could be organized and shared outside the traditional bureaucratic structure. The first draft of the portal was given shape on October 2, 2007. Shortly after, it was demonstrated to a group of desk officers, who embraced the idea and suggested ways that it could be further developed to better support their needs. One employee noted that it showed areas they didn't think of as their typical duties, which was helpful in giving them a broader sense of their responsibilities. Another discussed the value of providing links to information about support for their tasks: where she used to have to search the Department's directory to find the person who could help with forms related to an infrequent issue, now the information was available in a place instead of a person's head.

Portals in Wikipedia are usually created to support navigation of a multifaceted topic [24]. The structure represents a very small subset of the total Wikipedia article collection, and it can gather hundreds of pages to help readers understand a topic. The Diplopedia portal structure is very similar, but eDiplomacy staff also realized that personnel were interested in portals because they more closely mimicked typical web pages, and because they implied that there was more substance to a topic. Leveraging this success, eDiplomacy staff promoted the portal as a tool to help offices and posts to advertise their work to others with equities in their issues. If their information could be found, they'd be more likely to be invited to take part in the decision making around their areas of expertise.

\subsection{How Diplopedia Grew}

In State's initial efforts to ascertain the impact of Diplopedia on personnel and work practices, eDiplomacy staff tracked number of pages, contributors, edits, and page views of Diplopedia articles. While it is generally more difficult to ensure integrity of the page view tracking behind the firewall, staff swiftly noted that many more page views were occurring than registered contributors, article counts, or edits would indicate. This to some degree verifies the claim that Diplopedia activity is consistent with the Participation Inequality theory discussed above.

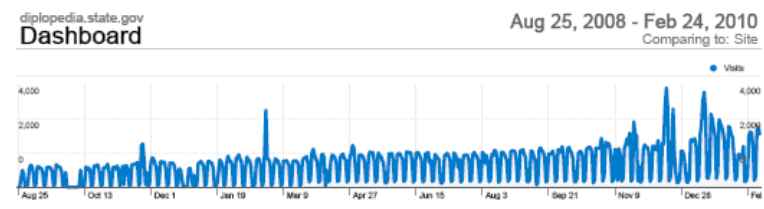

Figure 3. Diplopedia Utilization

From August 27, 2008 to February 20, 2010, average daily visits rose from $500-800$ to more than 2000. In reviewing the chart of these page views [25], there are frequent dips to indicate lower views on weekends and holidays, and occasional spikes based on times when the wiki was widely promoted, whether in the public or via Department Notices or cables. One of the days that saw the highest number of page views was January 14, 2010, when Department employees used Diplopedia to track the Haiti earthquake and recruit for task forces that supported the US Government response.

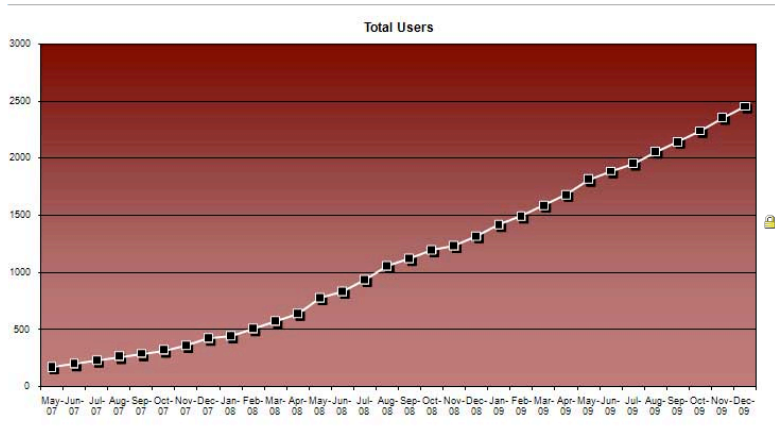

Figure 4. Total Users 
As the total articles and total contributors [26] climbed, so did page views, and most of this climb was consistent over time.

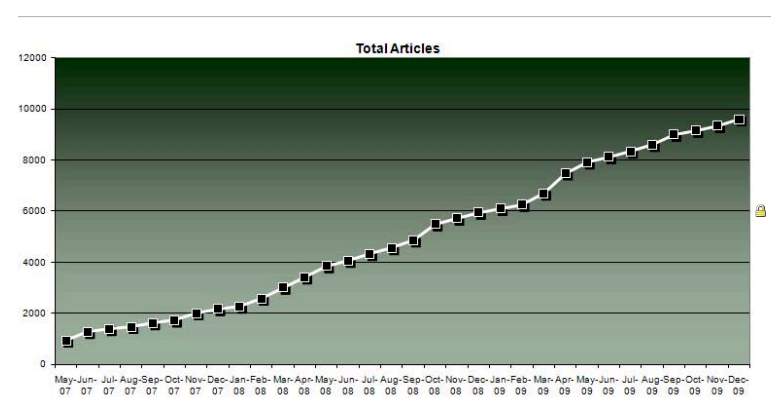

Figure 5. Total Articles

\section{GOING WIKI - OBSERVATIONS}

Building an enterprise wiki for the DoS came about because a group of eclectic but like-minded thinkers agreed that it would be of value to their organization. Tackling the orthodoxy on process in an agency where learning "how paper moves through the building," is still a requisite for employee effectiveness was not without its challenges, nor was it entirely quixotic. The coalition assembled to bring the MediaWiki software into the State Department tackled sacred cows regarding security, organizational process and skepticism regarding technology during the campaign to win approval.

In the end, the IT governance mechanism, the IT-CCB, worked. That said, a great deal of effort went into getting the blog and wiki software on the network. Much of that work was not in the business of software integration, but rather the job of organizational change. Making a small alteration in software acquisition process, was an enormous bureaucratic undertaking. This is a lesson that will have to be learned, and likely re-learned, on an agency-by-agency and office-by-office basis.

To do something radically different than accepted orthodoxy, the dyadic bureaucratic culture of the State Department had to be addressed. Run by appointees and managed by FSOs and Civil Servants, State shares many of the problems to be found in the Pentagon, where officers rise through the ranks and travel widely from assignment to assignment while a cohort of domesticallybased managers and experts is tasked with keeping much of the organization up and running. Having senior FSOs, both those on-duty, including eDiplomacy's office director, and retirees serving as consultants, backing the project was vitally important. At the same time, having State Department civil service staff of some rank, eDiplomacy's deputy, in the process was also enormously helpful. These managers, and their networks, provided a cohort of "godfathers" who could shepherd the project through bureaucratic rough patches.

A second item worthy of attention was the value of able contract staff. Having technologists who could make the case that a disruptive technological change could be good was enormously valuable. Without contract staff who could explain the pros and cons of different PHP versions or database inject security concerns, Diplopedia would have never seen the light of day.

Additionally, these factors were of importance in achieving the goal of producing a diplomacy wiki at the State Department:

- Recommendations from the 9/11 Commission and others for improved collaboration across agencies in response to the failures to connect information regarding terrorist activity gave bureaucratic cover for new ideas;

- Meme status of Wikipedia in 2005-2006 in U.S. popular culture and media meant that top-level leaders knew something about the technology;

- Recent hires under Secretary Powell's Diplomatic Readiness Initiative often had experience in technologically-sophisticated organizations;

- Secretary of State Rice's emphasis on Transformational Diplomacy was a natural rhetorical fit with unorthodox technological initiatives; and,

- No software was needed for installation on any users' personal computers.

In aggregate, these factors permitted the Office of eDiplomacy to deploy an enterprise wiki within 15 months of initiating the project. Further effort was required to engage employees and encourage them to contribute to Diplopedia over time, and the Project Lead position was established in order to have at least one person on staff who was committed full-time to ensuring both that the system was running and that employees were able to access and contribute to the wiki as they saw a value in doing so. .

Any collaborative technology effort will include multiple outputs. The first set encompasses those related to the network, infrastructure, and software deployments. The second has to do with the qualitative and quantitative assessments of the efficacy of the technology in providing a service. The outcomes from these activities continue to have an impact on the way the DoS conducts its

\footnotetext{
${ }^{9}$ This position, it should be noted, is not the "evangelist" position that other enterprise wikis have labeled it, although there is certainly some evangelizing required: instead, the Project Lead serves as the person who can report on the current state of being of Diplopedia at any given time and understands both the technology and Department processes well enough to see where the wiki might be of use to employees
} 
diplomatic work and collaborates within its employee base and throughout the Foreign Affairs community. Studies on collaborative technology deployment and development within other government agencies are limited, and further investigation of this area could provide further insight on how similar technology advances can change business processes throughout government.

\section{REFERENCES}

[1] C. Andrus, "The Wiki and the Blog," Studies in Intelligence Series, vol. 49 no.3.

[2] M. Burton, "Connecting the Virtual Dots," Studies in Intelligence Series, vol. 49 no. 3.

[3] C. Shannon, "A Mathematical Theory of Communication," Bell System Technical Journal, vol. 27, pp. 379-423, 623656, July, October, 1948.

[4] G. L. Argyros, M. Grossman, F. G. Rohatyn and A. Witkowsky, The Embassy of the Future, Washington, DC: CSIS, 2007, p. 19.

[5] Department of State, Foreign Affairs Handbook, 5-FAH-1 H-200, Telegrams, Aug. 1, 2007.

[6] P. Wait, "Jay Anania: State finds common ground in IT," Federal Computer Week, Sep. 21, 2005.

[7] J. Seigenthaler, "A false Wikipedia 'biography," USA Today, Nov. 29, 2005.

[8] M. J. Keegan, "Profiles in Leadership: Ambassador Patrick F. Kennedy-Managing Transformational Diplomacy," The Business of Government, IBM Center for the Business of Government, Spring 2008.

[9] C. Rice, "Remarks at Georgetown School of Foreign Service," Georgetown University, Washington, DC, Jan. 18, 2006.

[10] Y. Benkler, "Coase's Penguin, or Linux and the Nature of the Firm," The Yale Law Journal, 112 (3), 369-446, 2002.

[11] Office of Management and Budget, Circular A-130, Feb. 8, 1996.

[12] IEEE, IEEE Guide to Software Configuration Management, 1988.

[13] Department of State, Foreign Affairs Manual, 5-FAM-860 Hardware and Software Maintenance, Apr. 7, 2009.

[14] C. Thompson, “Open Source Spying," New York Times, Dec. 3, 2006.

[15] R. C. Bronk, "Diplopedia: Application of the Wiki Model for Collaborative Drafting in Foreign Affairs," in
Proceedings of Wikimania 2006, Cambridge, MA, Aug 4-6, 2006.

[16] N. Shachtman, "How to Restore Spies Credibility: Go Open Source," Danger Room [Online] Dec. 14, 2007. Available: http://www.wired.com/dangerroom/2007/12/how-torestore/

[17] R. Steele, The New Craft of Intelligence: Personal, Public, \& Political, Ashburn, VA: OSS, 2002.

[18] T. Smith Licciardi and L. Green, "Diplopedia Celebrates 10,000 Articles,” DipNote, Feb 4, 2009. Available: http://blogs.state.gov/index.php/site/entry/diplopedia_articl es

[19] About: Diplopedia page. Retrieved 4 January 2010 http://diplopedia.state.gov/index.php?title=About:Diploped ia

[20] D. Fletcher, "A Brief History of Wikipedia," Time, August $18,2009$.

[21] Decision Criteria for Tenure and Promotion in the Foreign Service. Available: http://www.afsa.org/mbr/CorePrecepts\%20\%20new\%202005.pdf

[22] M. Takahashi, M. Fujimoto, and N. Yamasaki, “Active Lurking: Enhancing the Value of In-House Online Communities Through the Related Practices Around the Online Communities", MIT Sloan Research Paper No. 4646-07; CCI Working Paper No. 2007-006, Apr 1, 2007.

[23] J. Nielsen. "Participation Inequality: Encouraging More Users to Contribute." 9 October 2006. Available: http://www.useit.com/alertbox/participation_inequality.htm 1

[24] Wikipedia Portal Article: http://en.wikipedia.org/wiki/Wikipedia:Portal

[25] Image courtesy Google Analytics, https://www.google.com/analytics/reporting/?reset=1\&id=9 665258

[26] Charts of Diplopedia Total Users and Total Articles, http://diplopedia.state.gov/index.php?title=Archive_Statisti cs 